\title{
An animal by any other name : identité culturelle et symbolisme animal dans le Roméo et Juliette d'Oh Tae-Suk
}

An Animal by Any Other Name: Cultural Identity and Animal Symbolism in Oh Tae-Suk's Romeo and Juliet

\section{Charlène Cruxent}

\section{(c) OpenEdition \\ Journals}

\section{Édition électronique}

URL : http://journals.openedition.org/shakespeare/5672

DOI : $10.4000 /$ shakespeare. 5672

ISSN : 2271-6424

Éditeur

Société Française Shakespeare

\section{Référence électronique}

Charlène Cruxent, «An animal by any other name : identité culturelle et symbolisme animal dans le Roméo et Juliette d'Oh Tae-Suk », Actes des congrès de la Société française Shakespeare [En ligne], 38 2020, mis en ligne le 26 juin 2020, consulté le 06 juillet 2020. URL : http://journals.openedition.org/ shakespeare/5672; DOI : https://doi.org/10.4000/shakespeare.5672

Ce document a été généré automatiquement le 6 juillet 2020.

(C) SFS 


\title{
An animal by any other name : identité culturelle et symbolisme animal dans le Roméo et Juliette d'Oh Tae-Suk
}

\author{
An Animal by Any Other Name: Cultural Identity and Animal Symbolism in Oh \\ Tae-Suk's Romeo and Juliet
}

Charlène Cruxent

\section{Introduction}

1 Oh Tae-Suk est un dramaturge et metteur en scène sud-coréen connu pour ses mises en scène hautes en couleur mêlant danse et chant. Le traitement particulier du temps et de l'espace, ainsi que certaines omissions par rapport aux textes qu'Oh Tae-Suk adapte, demande une participation active du spectateur que le metteur en scène cherche à obtenir. Depuis ses débuts sur la scène artistique dans les années 1960, il n'a cessé d'expérimenter de nouvelles techniques pour trouver sa propre esthétique théâtrale, ce qui s'est traduit par des changements constants au niveau du texte et/ou de la scénographie lors des représentations d'une même pièce. Oh Tae-Suk a mis en scène une œuvre de Shakespeare pour la première fois en 1995 et son choix s'est porté sur Roméo et Juliette. L'histoire d'un amour impossible entre deux jeunes gens appartenant à des clans opposés donne à voir un antagonisme qu'Oh Tae-Suk révèle et intensifie dans sa mise en scène de Roméo et Juliette. Le public ayant assisté à la représentation par la compagnie théâtrale sud-coréenne Mokwha Repertory Company le 26 avril 2018 au théâtral national de Craiova en Roumanie, a pu s'en apercevoir, et ce avant même que la représentation ne commence. En entrant dans l'auditorium, les spectateurs constatent la présence d'une toile au milieu de la scène, toile sur laquelle figure le dessin d'une tortue et d'un serpent entremêlés ${ }^{1}$. Ces deux animaux représentent Hyeon$m u$ (사신도(현무), aussi connu sous le nom de Tortue-Serpent, figure de la mythologie 
coréenne qui était responsable du maintien de l'harmonie entre le yin et le yang, deux forces cosmiques opposées ${ }^{2}$.

La représentation commence avec le retentissement du tonnerre, le retrait de la toile et l'entrée d'une quinzaine d'acteurs. Deux groupes distincts s'affrontent : munis d'épées, les acteurs exécutent une chorégraphie au ralenti. Ils font alors des mouvements semblables à ceux effectués lors d'un combat d'art martial. L'harmonie et la quiétude présentes jusqu'alors sur scène disparaissent donc au moment même où la toile représentant Hyeon-mu, la Tortue-Serpent, est retirée de la scène. Symboliquement, cet animal mythique et mystique véhicule un sens qu'un spectateur pourrait occulter s'il n'a pas connaissance de sa signification. En effet, la pièce est jouée en coréen et si une traduction en anglais est fournie via des écrans au-dessus de la scène, aucune indication n'est affichée pour exposer l'identité d'Hyeon-mu.

3 Tout au long de la représentation, pléthore de références aux animaux, tel le renard, apparaissent. De la même façon que pour la Tortue-Serpent, elles ne peuvent être comprises dans leur globalité que par le truchement du folklore coréen, ce qui a poussé plusieurs critiques de théâtre à considérer la mise en scène d'Oh Tae-Suk comme un " Romeo and Juliet Korean Style ${ }^{3}$ ". L'utilisation du symbolisme animal dans le Roméo et Juliette du dramaturge témoigne en effet de l'appropriation de la tragédie shakespearienne dans un contexte coréen, mais pas seulement. Les changements opérés par le metteur en scène entre la première représentation de son Roméo et Juliette en 1995 et celles des années 2010 montrent qu'Oh Tae-Suk, bien qu'il utilise des éléments typiques du théâtre coréen, présente le mythe des amants maudits comme universel. La présence de certains animaux lors de la représentation de 2018 ajoute d'ailleurs une dimension optimiste et positive à la tragédie shakespearienne avec l'espoir d'une paix universelle à l'échelle nationale (réunification de la Corée, nation qui fut scindée en deux à la fin de la Seconde Guerre Mondiale) et mondiale (d'où le choix d'exporter la représentation théâtrale en dehors de la Corée).

\section{Appeler un chat un chat : caractérisation animale et folklore coréen}

\section{Des noms et des masques}

4 La scène de combat qui marque le début de la représentation révèle les tensions entre deux familles opposées : un groupe de jeunes gens portant des habits verts (clan des Montaigu) et un autre revêtant des costumes orange (clan des Capulet) font irruption sur scène, agitent leur joy - une arme en bois - exécutant ainsi ce qui s'apparente à une chorégraphie Geommu, ou danse de l'épée traditionnelle en Corée. Après cette scène d'affrontement, la nourrice fait son apparition et apporte une note d'humour pour alléger l'atmosphère tendue - le mélange de la tragédie et de la comédie est un motif récurrent dans le Roméo et Juliette d'Oh Tae-Suk. Elle arrive en courant, interpellant les personnes présentes en utilisant des noms d'animaux : «Blaireau ! Grenouille ! Lézard ! Raton laveur! Pieuvre! Sauterelle!» («Badger! Frog! Lizard! Raccoon! Octopus! Grasshopper! $\left.{ }^{4} »\right)$. Cette ouverture n'a nullement surpris Kim Yun-Cheol, directeur artistique du «National Theater Company of Korea » et président de l'Association Internationale des Critiques de Théâtre, qui explique que dans la culture coréenne, chaque animal est associé à une émotion ou un trait de caractère humain ${ }^{5}$. Les 
personnages de la tragédie shakespearienne ne correspondent donc pas à un nom propre, mais à un ou plusieurs attribut(s) conféré(s) à chaque animal. Kim Yun-Cheol indique que les Coréens se donnent souvent des noms d'animaux entre eux en fonction de ce qu'ils veulent exprimer ou dire sur la personne qu'ils interpellent ou surnomment. En outre, il attire l'attention sur les masques d'animaux que certains acteurs portent dans la mise en scène d'Oh Tae-Suk car ils reflètent une caractéristique intrinsèque du personnage, renseignant ainsi les spectateurs sur son identité mais également sur ses intentions et son destin.

\section{Les amants maudits : animaux et influence du destin, ou le rôle de l'astrologie}

Dans Chinese Astrology: Exploring the Eastern Zodiac (2005), Shelly Wu révèle que les signes $\mathrm{du}$ zodiaque chinois définissent une personne en fonction de l'animal qui lui est associé ${ }^{6}$. L'importance donnée par Kim Yun-Cheol aux masques dans la mise en scène d'Oh Tae-Suk peut faire penser à l'influence de l'astrologie chinoise qui a une place considérable dans la culture coréenne. L'ajout de ces accessoires devrait donc éclairer le spectateur quant à l'identité des personnages. Lorsque Roméo et son entourage se préparent pour aller au bal organisé par les Capulet, Mercutio fait part de l'idée de se déguiser en fonction des signes du zodiaque pour ne pas être reconnus ( We shall mask ourselves with the signs of the Zodiac $\left.{ }^{7} »\right)$. Les jeunes gens du clan des Montaigu arborent donc des masques de cheval, chèvre, chien ou mouton ${ }^{8}$.

Mercutio choisit le cochon comme emblème personnel ("I shall be the pig $\left.{ }^{9}\right)^{10}$, signe du zodiaque associé à l'honnêteté, la liberté, une certaine tendance à l'égoïsme et une aversion contre l'injuste ${ }^{11}$. Ces caractéristiques semblent bien désigner ce personnage dont le nom propre n'est pas mentionné dans la mise en scène d'Oh Tae-Suk-comme l'équivalent de Mercutio dans le Roméo et Juliette de Shakespeare. L'importance accordée à l'astrologie chinoise n'est pas seulement d'ordre pratique, car si les signes du zodiaque permettent de caractériser de façon archétypale un personnage véhiculant les traits de personnalité et le tempérament associés à tel ou tel animal, ils sont fondés sur un calcul mathématique prenant en compte la position des étoiles et planètes lors de la naissance d'un individu ${ }^{12}$. Les étoiles occupent donc une place prépondérante, et en fonction de l'évolution de ces dernières dans le ciel, elles peuvent avoir une influence sur le présent et le futur des différents signes du zodiaque. Mercutio fait remarquer ceci lorsqu'il demande à Tybalt de reporter leur duel car l'orientation des étoiles ne semble pas être favorable à une personne native du cochon ce jour-là (« Let's fight tomorrow, today is not a good day according to my zodiac sign $\left.{ }^{13} »\right)$.

7 Oh Tae-Suk reprend ici le paradigme déjà présent dans la tragédie shakespearienne selon lequel la position des astres dans le ciel a une influence sur les individus. Roméo et Juliette sont définis dans le prologue de la pièce comme des amants maudits ("starcrossed lovers ${ }^{14}$ ", Romeo and Juliet prologue 1.6), c'est-à-dire deux personnes subissant l'alignement de planètes qui exercent une influence malveillante ${ }^{15}$. Même si Roméo déclare vouloir s'affranchir du joug des astres ("Then I defy you, stars ", Romeo and Juliet, V.i.24), il n'échappe pas à son triste sort. Cette corrélation entre les planètes et la vie terrestre n'est d'ailleurs pas une idiosyncrasie de Shakespeare. L'astrologie était communément considérée comme une science durant la période élisabéthaine, et l'équivalent de ce que nous connaissons sous le nom d'horoscope était calculé de façon 
à comprendre la personnalité et le destin de chaque individu ${ }^{16}$. La reine Élisabeth $1^{\text {re }}$ avait recours aux services de John Dee, mathématicien et astrologue diplômé de l'Université de Cambridge, qui lui indiquait le moment propice pour lancer une attaque ou organiser des événements importants ${ }^{17}$.

\section{Emblème animal}

8 Dans la mise en scène d'Oh Tae-Suk d'avril 2018, Juliette n'est pas singularisée par le biais d'un signe car elle ne porte pas de masque. Cependant, elle est tout de même associée, de la même manière que Mercutio, à un animal - le renard. Lors de la scène du bal, alors que les jeunes hommes du clan des Montaigu portent des masques, les demoiselles du clan des Capulet, vêtues de blanc, tiennent des panneaux rouges de façon à former la silhouette d'un renard $^{18}$. La couleur rouge crée ici un rappel chromatique et lie Mercutio aux pancartes, comme si ce dernier pouvait aider le spectateur à comprendre l'image du renard en identifiant le lien entre la caractérisation animale via les masques/signes du zodiaque et la présence du renard entre les mains des jeunes femmes. Dans le bestiaire coréen, le renard blanc à neuf queues est vu comme un signe de bon augure en temps de paix, mais c'est surtout l'image du renard comme charmeur qui prévaut dans la mise en scène d'Oh Tae-Suk. En coréen, le mot pour désigner un renard, yeo-u (여우), fait aussi référence à une jeune femme qui sait comment agir afin d'obtenir ce qu'elle veut ${ }^{19}$. Le renard est en effet associé à des figures féminines qui font bon usage de leur charme et leur intelligence pour attirer leur partenaire (ou leur proie, en fonction du contexte). C'est le cas de Juliette qui flirte ouvertement avec Roméo lors de la scène du bal et (se) joue de/avec lui lorsqu'il rejoint sa nouvelle épouse dans la chambre de cette dernière. Recouverte d'un tissu blanc, la scène devient un gigantesque lit dans lequel les amoureux folâtrent ${ }^{20}$. Utilisant ce qui fait office de linge de lit, Juliette joue à cache-cache avec son mari, jeu qui a pour résultat de prendre au piège Roméo qui se retrouve, telle une momie, coincé dans les draps de sa bien-aimée.

\section{Un Roméo et Juliette universel : l'esthétique d'Oh Tae- Suk, d'est en ouest}

\section{L'amour donne des ailes : absence de (petits) noms}

9 Même si des différences par rapport au texte source apparaissent par le biais du symbolisme animal coréen en ce qui concerne l'identification et la caractérisation des personnages, plusieurs éléments peuvent nous amener à voir ce Roméo et Juliette comme une représentation universelle d'une histoire d'amour impossible. Tout d'abord, les deux familles qui s'affrontent dans la mise en scène d'Oh Tae-Suk ne sont à aucun moment identifiées par leur nom. Le directeur artistique du «National Theatre of Korea » a fait remarquer que même si les patronymes "Montaigu» et "Capulet » étaient retranscrits sur les écrans au-dessus de la scène où une traduction en anglais était affichée, les noms de famille n'étaient pas inclus dans le script coréen. Les deux clans opposés peuvent cependant être distingués grâce aux couleurs de leur tenue, vert et bleu ou orange et marron, en fonction de leur affiliation au camp des Capulet ou des Montaigu. De la même façon, les prénoms des divers personnages ne sont pas 
mentionnés, ce qui donne à l'injonction de Roméo « Let us both abandon our names and leave ${ }^{21} »$ une dimension universelle.

Parallèlement, les noms affectueux échangés entre Roméo et Juliette lors de la scène du balcon ne sont pas cités ou traduits en coréen. Ils sont cependant suggérés à travers, entre autres, des effets sonores. En effet, la scène est ponctuée de chants d'oiseaux, ce qui rappelle l'appellation affectueuse «mon tout petit faucon ${ }^{22}$ » («my nyas ", Romeo and Juliet, II.ii.168). La dimension visuelle est également exploitée pour véhiculer les sentiments amoureux des personnages. D'une part, le surnom de Juliette est retranscrit dans les gestes de cette dernière lorsqu'elle compare indirectement ses bras et son corps à une cage dans laquelle elle envelopperait son prétendant :

ROMEO I'm here.

JULIET Don't go too far. You are a bird in my hand.

With a silk thread I pluck you back.

ROMEO Where's the cage?

JULIET Here.

(The two dance together.) ${ }^{23}$

11 Après avoir embrassé Roméo pour la première fois lors de la scène du bal, Juliette dit que Roméo est son oiseau. Cette phrase est ensuite suivie par des chants d'oiseaux et une chorégraphie des deux acteurs pendant laquelle ils dansent et imitent le vol des oiseaux.

D'autre part, la toile visible sur scène au début de la représentation sur laquelle figure Hyeon-mu (ou la Tortue-Serpent), symbole de l'harmonie, réapparait lorsque Roméo et Juliette se retrouvent lors de la scène du balcon. Cette union entre le yin (principe masculin) et le yang (principe féminin), deux forces cosmiques qui s'opposent mais sont complémentaires, est l'incarnation visuelle des deux jeunes gens : ils appartiennent à des familles différentes, mais leur association aurait pu amener à un accord et une cohésion de la société dans laquelle ils évoluent, mettant ainsi fin à l'antagonisme entre les factions divergentes. Ceci est également sous-entendu à travers le masque de Roméo qui représente un cheval ${ }^{24}$, se rapprochant ainsi de la jeune Capulet dont le nom de famille peut dériver de « capel », forme prémoderne voulant dire « cheval ${ }^{25}$ ». Le jeune couple n'est donc pas identifié comme "Roméo » et "Juliette ", non seulement parce que ces noms ne sont pas présents dans la mise en scène coréenne, mais aussi parce qu'Oh Tae-Suk fait appel à des concepts qui évoquent les sentiments amoureux à travers l'image des oiseaux qui roucoulent, sans jamais nommer les individus concernés.

\section{Jeux de mains, jeux de vilains : une jeunesse innocente}

13 L'histoire tragique des amants maudits est expliquée dans cette représentation par leur immaturité; ils sont représentés comme des enfants ou adolescents naïfs qui ne comprennent pas les événements qui se produisent autour d'eux. Si de nombreux metteurs en scène interprètent également Roméo et Juliette comme une tragédie de l'adolescence, Oh Tae-Suk met en exergue la puérilité des personnages par le biais d'un jeu impliquant des animaux. En effet, Tybalt est abasourdi par les proportions qu'ont prises les plaisanteries avec Mercutio. Ils commencent par jouer à un jeu appelé « crapaud et taupe» (« to play toad and mole $\left.{ }^{26} »\right)$, qui consiste à identifier des formes d'animaux dans les nuages. 
TYBALT Mercutio, thou consortest with Romeo.

What does that cloud look like?

MERCUTIO A mole.

TYBALT No, it looks like a toad. Like you.

MERCUTIO No, it's a mole. Like you!

TYBALT Is it a toad or a mole?

YOUNG CAPULETS A toad!

MERCUTIO Stop! Here's that shall make you dance.

Open your arms, bend your knees, lift up your chin.

ALL Mole, toad, salmon, trout, carp, half-moon - (they sing)

PASSING LADY It's so hot weather! Do you feel like singing? Well, anyway, it's better

than fight. Mole, toad, salmon. ${ }^{27}$

14 Mais le ton monte vite entre Tybalt et Mercutio et la situation dégénère. Un combat éclate, combat qui est joué au ralenti et au cours duquel l'une des techniques employées consiste à chatouiller l'adversaire ${ }^{28}$. Le conflit se termine par le décès de Mercutio. Tybalt se recroqueville sur lui-même ${ }^{29}$, pleurant une mort qu'il a lui-même infligée. Il se justifie en expliquant qu'il voulait simplement s'amuser et que la faute incombe au défunt car ce dernier avait commencé les hostilités («I'm hot. What's with this weather? / We were only playing. Serves him right. / He drew first, you saw it. Kinsman, you saw it too $\left.{ }^{30} »\right)$. Le sang de son ami sur les mains, Roméo sanglote avant de poignarder Tybalt.

\section{Ouvrez la cage aux oiseaux : l'échec des autorités et le poids des générations passées}

15 La présence de chaînes autour du bras des adversaires peut faire penser au poids que représentent les autorités (voir note 26), empêchant les oisillons - la nouvelle génération - de voler de leurs propres ailes. Ces jeunes gens ne se battent pas par haine : ils sont victimes d'une histoire que leur famille leur impose, d'un passé que les figures dirigeantes ne semblent pas pouvoir contrôler. La figure du prince est tournée en dérision dès le début de la représentation. Même s'il est grand et qu'il porte un imposant costume jaune ${ }^{31}$, Mercutio se moque de lui en l'imitant lorsqu'il entre en scène habillé en femme et répète les mots du prince, ce qui a pour effet de déclencher le rire des personnes assistant à cette parodie. Après la mort de Roméo et Juliette, le prince avoue qu'il a manqué à son rôle et demande à être sacrifié. Selon Byung Yong Lee, agent de la compagnie théâtrale Mokwha Repertory Company et acteur qui campe le rôle du prince, cette figure prend de la place sur scène afin de combler son manque d'autorité ${ }^{32}$. Il est tel un épouvantail : visuellement impressionnant mais impuissant.

Lord Capulet et Lord Montaigu ne sont que rarement sur scène, et la nourrice est quant à elle un piètre soutien pour la jeune Capulet. Non seulement elle pleure et doit être réconfortée par Juliette lorsqu'elle apprend la disparition de Tybalt, mais son penchant pour l'alcool ne fait pas d'elle une personne digne de confiance pour élever une adolescente $^{33}$. De plus, elle est appelée « Milan Royal» (« red kite $\left.{ }^{34} »\right)$ et tient un oiseau empaillé lors de son entrée en scène au début de la représentation ${ }^{35}$. La référence à ce rapace diurne, en plus du kimono rouge que la nourrice porte et qui donne l'impression qu'elle vole sur scène, peut faire penser à chujak-ki, ou l'oiseau rouge du sud. Cette créature mythique ne présage rien de bon car elle était présente sur les drapeaux coréens au $\mathrm{XX}^{\mathrm{e}}$ siècle en temps de guerre ${ }^{36}$. 
monde des adultes n'agissent pas pour arrêter le conflit historique entre les deux clans. Ces deux clans peuvent évoquer la situation en Corée : cette nation fut scindée en deux à la fin de la Seconde Guerre Mondiale et le conflit armé qui opposa la Corée du Nord et celle du Sud (1950-1953) ne permit aucune réunification du territoire. Les relations intercoréennes sont en effet chères à Oh Tae-Suk, mais le metteur en scène semble également mener une réflexion plus globale sur les antagonismes présents dans les diverses parties du monde. L'agent de la compagnie théâtrale Mokwha Repertory Company, Byung Yong Lee, fait part de la dimension universelle qu'Oh Tae-Suk a développée ces dernières années. Il explique que les individus, malgré leur différence physique, sont tous semblables dans la mesure où ils aspirent à la paix ${ }^{37}$. Les dernières minutes de la mise en scène peuvent d'ailleurs laisser entrevoir une lueur d'espoir pour cette conciliation tant désirée.

\section{S'envoler vers de nouveaux horizons : espoir universel et international}

\section{Quand les murs tremblent}

18 Il semble donc que l'adaptation de Roméo et Juliette par Oh Tae-Suk puisse être interprétée comme une reconnaissance universelle de l'échec des autorités à imposer ou construire une paix pour les nouvelles générations. L'évolution des représentations de son Roméo et Juliette montre bien que le metteur en scène a cherché à faire passer un message au-delà de la Corée. Les premières représentations (fin des années 1990) restaient très proches du texte de Shakespeare. En 2001, plusieurs éléments de la culture coréenne ont été ajoutés et à partir de 2013, la fin de la mise en scène a été altérée de manière drastique. En effet, ce ne sont plus seulement les deux amants qui meurent, mais tous les personnages sur scène ${ }^{38}$, montrant ainsi combien il est important de régler les conflits passés car leur impact ne se fait pas seulement ressentir sur un petit nombre d'individus, mais sur la société toute entière.

En 2017, un nouvel élément est également apparu: le mur en papier mâché qui constitue les enceintes de la ville sur scène est détruit. Ce mur a une fonction symbolique forte pour Oh Tae-Suk : rapportant les paroles de ce dernier, les acteurs de Mokwha Repertory Company expliquent que Tae-Suk a envié les Allemands en 1989 lorsque le mur de Berlin a été démoli, permettant ainsi à de nombreuses familles de retrouver un être cher. Alors qu'il était enfant, le metteur en scène est rentré chez lui un soir pour découvrir que son père avait été enlevé et déporté en Corée du Nord. Il n'a plus jamais revu ce dernier, et la situation en Allemagne en 1989 l'a donc énormément touché. ${ }^{39}$ Dans ce chaos qui envahit la scène lorsque le mur de papier mâché tombe, il est également possible de voir une référence à l'ambition du président américain nouvellement élu en 2017, Donald Trump, de construire un mur entre les États-Unis et le Mexique, et l'espoir d'Oh Tae-Suk que ce mur connaisse la même fin que celui de Berlin $^{40}$. 


\section{Sortir de la cage : émancipation de la nouvelle génération} une touche d'optimisme survient dans les derniers instants de la représentation. Après l'effondrement du mur, le public se met à applaudir car l'atmosphère sombre semble correspondre à la fin de la tragédie shakespearienne. Cependant, une lumière verte persiste sur scène, mettant ainsi en exergue la présence d'un buisson fait de lierre et de lianes accroché sur le seul pan du mur restant sur scène $e^{41}$. Après quelques secondes, deux points lumineux émanent de ce buisson végétal, comparables à deux lucioles qui s'envolent hors de scène. Ces lucioles sont une réincarnation de Roméo et Juliette voulant échapper à leur destin. Le choix de ces insectes volants, les lucioles, fait aussi penser au mythe de Liang-Zhu, ou les "amants papillons». Cette légende populaire chinoise, qui a commencé à circuler en Corée au $7^{\mathrm{e}}$ siècle, raconte l'histoire d'amour impossible entre Liang Shanbo et Zhu Yingtai. Les parents de Zhu ayant choisi un époux pour cette dernière, Liang se laisse mourir car il est conscient qu'il ne sera jamais réuni avec celle qu'il aime. Après la cérémonie de mariage, lorsque Zhu se rend compte de la mort de son bien-aimé devant sa tombe, elle demande à le rejoindre. Le tombeau s'ouvre alors et Zhu s'y précipite. D'après la légende, deux papillons sortirent de la sépulture, s'envolant ensemble vers l'infini. De manière similaire au Roméo et Juliette de Shakespeare, Oh Tae-Suk semble s'approprier et adapter le récit chinois dans sa mise en scène afin d'attirer l'attention sur une autre question contemporaine qui le préoccupe : le respect de l'environnement.

\section{Une lueur d'espoir : lorsque l'environnement reflète l'état de la société}

21 Le choix des lucioles - et non pas des papillons - est interprété par Byung Yong Lee, l'agent de Mokwha Repertory Company, de la façon suivante: ces insectes sont des animaux qui évoluent là où l'air est pur et sain, le même genre d'environnement dans lequel les nouvelles générations pourraient créer leur propre histoire et voler de leurs propres ailes en prenant en compte et en respectant la nature. ${ }^{42}$ Il continue en disant que le mur de briques représente le monde industriel que les adultes et les générations précédentes ont érigé, et sa destruction montre que ce modèle n'est pas (ou plus) viable. Les lucioles symbolisent un changement, une transition dans un environnent sain qui n'est possible que par le biais des nouvelles générations qui doivent se rappeler leur passé afin d'initier une nouvelle sociétée ${ }^{43}$. Lors de la représentation de Roméo et Juliette en avril 2018, la présence du mur, des lucioles ainsi que de sirènes d'ambulance qui retentissent, incarnent ce passé et les affrontements douloureux qui le caractérisent. Ces trois éléments peuvent d'ailleurs suggérer l'influence de l'esthétique déployée dans le film Le tombeau des lucioles (1988) d'Isao Takahata ${ }^{44}$. Bien que l'intrigue se déroule au Japon (et non en Corée), le film narre l'histoire de deux jeunes adolescents qui périssent à la suite du conflit de la Seconde Guerre Mondiale. Malgré la disparition de leurs corps, les esprits des jeunes gens demeurent intacts : ils évoluent parmi les lucioles présentes sur la montagne surplombant la ville de Kobe, observant ainsi la reconstruction lente de la cité après la fin de la guerre. D'après Byung Yong Lee, le motif des lucioles dans la représentation n'est pas anodin : au croisement du mythe chinois des "amants papillons » et de la croyance japonaise selon laquelle l'âme des défunts s'incarne dans un animal, les lucioles ne peuvent exister que si

Actes des congrès de la Société française Shakespeare, 38 | 2020 
l'environnement dans lequel elles évoluent est dépourvu de pollution. ${ }^{45}$ Dans la mise en scène, cette pollution transparaît de façon littérale à travers l'importance qui est accordée à la nature, et de façon métaphorique, par le biais des relations tendues entre les hommes qui font obstacle à l'épanouissement de la nouvelle génération.

\section{Conclusion}

Hyeon-mu, ou la Tortue-Serpent, figure de la mythologie coréenne responsable du maintien de l'harmonie dans la société ne réapparaît pas à la fin de la représentation. Non pas parce que cette harmonie ne peut pas être atteinte, mais parce que la nouvelle génération qui est représentée par Roméo et Juliette - ou plutôt par les deux amants anonymes associés aux chants des oiseaux et aux deux lucioles qui s'envolent vers de nouveaux horizons - doit construire un nouvel équilibre en gardant à l'esprit les conflits passés pour mieux aller de l'avant. La dernière scène, dont l'interprétation est laissée au spectateur, a d'ailleurs pris une nouvelle dimension le lendemain de la représentation à Craiova. Kim Jong-un et Moon Jae-in, les dirigeants respectifs des deux Corées, ont franchi la zone démilitarisée se trouvant entre la Corée du Nord et celle du Sud et se sont rencontrés afin d'entamer une procédure pour dénucléariser la péninsule coréenne et mettre ainsi fin à la guerre entre les deux pays. Cette rencontre intercoréenne, même si elle s'inscrit dans un contexte sociopolitique international complexe, pourrait être vue à travers le prisme des lucioles dans la mise en scène d'Oh Tae-Suk: c'est un évènement qui a donné l'impression qu'une réconciliation était possible ${ }^{46}$. Bien sûr, il reste un long chemin à parcourir avant que cette lueur d'espoir ne se concrétise et donne place à une entente sur le long terme, en Corée ou ailleurs. Oh Tae-Suk encourage et met en scène de façon subtile dans son adaptation de l'histoire tragique des amants maudits cette espérance grâce notamment au symbolisme animal. Le metteur en scène emprunte à Shakespeare (faucon, cheval, signes du zodiaque européen) et au folklore coréen (Tortue-Serpent, renard, signe du zodiaque chinois, milan royal, lucioles) des éléments animaliers qu'il exploite et remanie pour se les approprier. Oh Tae-Suk crée son Roméo et Juliette qui, bien que sanglant, diffuse l'espoir d'une paix entre les peuples (et non pas seulement entre deux clans) et d'une cohésion entre les êtres humains, les animaux et la nature. La scène finale (pan de mur recouvert de lierre d'où les lucioles émanent), qui est absente dans le texte source, est peut-être une façon d'interpeller les spectateurs : il ne faut pas oublier les conflits qu'il y a eu dans le passé car ils font partie de notre histoire (tout comme les œuvres de Shakespeare), mais il faut les transcender et aller plus loin que nos prédécesseurs pour tendre vers une harmonie universelle.

\section{NOTES}

1. Plusieurs photographies prises lors de la représentation sont disponibles sur le profil Facebook «Festivalul International Shakespeare, Craiova» (documents mis en ligne le 27 avril 2018), 
accessible en ligne sur: https://www.facebook.com/pg/shakespearefestivalcraiova/photos/? ref=page_internal.

La photographie de la toile est d'ailleurs accessible en ligne sur : https://www.facebook.com/ shakespearefestivalcraiova/photos/a.2042982645953542/2042996879285452/?type=3\&theater, consulté le 30 janvier 2020.

2. Musée National de Corée, Exposition «Tomb Murals of the Four Guardian Deities from Gangseodaemyo ", accessible en ligne sur: https:/www.museum.go.kr/site/eng/ exhiSpecialTheme/view/specialGallery?exhiSpThemId=4179\&listType=list, consulté le 07 décembre 2018.

3. Saffron Vicky Walker, "'Romeo and Juliet' Korean style", 28 juillet 2010, https:// saffronatstudy.wordpress.com/2010/07/28/romeo-and-juliet-korean-style/, consulté le 10 décembre 2018.

4. Transcription (partielle) de la représentation de Roméo et Juliette (2006), MIT Global Shakespeare, https://globalshakespeares.mit.edu/wp-content/uploads/romeo-and-juliet-oh-taesuk-2006-script-1.pdf, consulté le 15 mai 2019, p. 1.

5. Kim Yun-Cheol, directeur artistique du "National Theater Company of Korea", communication personnelle, 27 avril 2018, Craiova.

6. Shelly Wu, Chinese Astrology: Exploring the Eastern Zodiac, The Career Press, Franklin Lakes, 2005, p. 14. Le zodiaque chinois comprend douze animaux : le Rat, le Bœuf, le Tigre, le Lapin, le Dragon, le Serpent, le Cheval, la Chèvre, le Singe, le Coq, le Chien et le Cochon.

7. Transcription de la représentation de Roméo et Juliette (2006), op. cit., p. 3.

8. Simon Buia, «Romanian Actors", photographie accessible en ligne sur: http:// www.romanianactors.com/wp-content/uploads/2018/04/ROMEO-\%C5\%9EI-JULIETA-MokwhaRepertory-Company-din-Seoul-Coreea-de-Sud-regia-Oh-Tae-Suk-Photographer-Simion-Buiawww.romanianactors.com-4280048.jpg, consulté le 30 janvier 2020.

9. Transcription de la représentation de Roméo et Juliette (2006), op. cit., p. 3.

10. Simon Buia, op.cit., http://www.romanianactors.com/wp-content/uploads/2018/04/ROMEO\%C5\%9EI-JULIETA-Mokwha-Repertory-Company-din-Seoul-Coreea-de-Sud-regia-Oh-Tae-SukPhotographer-Simion-Buia-www.romanianactors.com-4280052.jpg, consulté le 30 janvier 2020.

11. "In Korea, each animal has a certain character. We characterize each animal in term of human emotions or human characters. 'Pig' could be greedy; 'snake' could be very sly, etc. » Kim Yun-Cheol, communication personnelle, op. cit.

12. Shelly Wu, op. cit., p. 11.

Dans ce sens l'astrologie chinoise est similaire à l'astrologie européenne. C'est la nature des signes qui différe.

13. Transcription de la représentation de Roméo et Juliette (2006), op. cit., p. 3.

14. William Shakespeare, Romeo and Juliet, éd. René Weis, coll. The Arden Shakespeare, Londres, Bloomsbury, 2012, p. 123. Toutes les citations tirées de Romeo and Juliet proviennent de cette édition.

15. L'astrologue Priscilla Costello indique que l'impulsivité excessive joue un rôle décisif dans l'intrigue de Roméo et Juliette, et que cette impétuosité s'explique par l'influence négative de Mercure.

Priscilla Costello, Romeo and Juliet: The Hidden Astrological Keys, Lake Worth, Ibis Press, 2018, p. 36-7.

16. Lauren Kassell, Michael Hawkins, Robert Ralley et John Young, «Early modern astrology ", dans A Critical Introduction to the Casebooks of Simon Forman and Richard Napier, 1596-1634, accessible en ligne sur: https://casebooks.lib.cam.ac.uk/astrological-medicine/early-modernastrology, consulté le 24 janvier 2020.

17. Idem et J. W. Draper, "Shakespeare's 'Star-Crossed Lovers' ", The Review of English Studies 15.57, Jan., 1939, 16-34, p. 20. 
Pour plus de détails concernant l'astrologie pendant la Renaissance, consulter Brendan Dooley (éd.), A Companion to Astrology in the Renaissance, Leiden et Boston, Brill, 2014.

18. Festivalul International Shakespeare, Craiova, Facebook, photographie accessible en ligne sur : https://www.facebook.com/shakespearefestivalcraiova/photos/a.

2042982645953542/2042994859285654/?type=3\&theater, consulté le 30 janvier 2020.

19. Richard E. Strassberg, A Chinese Bestiary: Strange Creatures from The Guideways Through Mountains and Seas, Berkley, University of California Press, 2002, p. 88.

20. Festivalul International Shakespeare, Craiova, op. cit., https://scontent-mrs2-1.xx.fbcdn.net/ v/t31.0-8/30848332_2042996185952188_3281415614556735402_o.jpg?

_nc_cat $=105 \&$ _nc_ohc=WYUuTsUAcHMAX-hQcZ1\&_nc_ht=scontent-

mrs2-1.xx\&oh=819dff0d8cbe9ef46dbb9a781c483481\&oe=5ED8A51B, consulté le 30 janvier 2020.

21. Scène du balcon. Réplique affichée sur les écrans au-dessus de la scène lors de la représentation du 26 avril 2018 dans le théâtre national de Craiova.

22. William Shakespeare, éd. et trad. Jean-Michel Déprats et Gisèle Venet, Tragédies I (Cuvres complètes Shakespeare I), Gallimard, Paris, 2002, p. 281.

23. Transcription de la représentation de Roméo et Juliette (2006), op. cit., p. 12.

24. Festivalul International Shakespeare, Craiova, op. cit., https://scontent-mrs2-1.xx.fbcdn.net/ v/t31.0-8/30171349_2042997392618734_328852707667876469_o.jpg?

_nc_cat=103\&_nc_ohc=hw5i1jW5GJ4AX-zO68Z\&_nc_ht=scontentmrs2-1.xx\&oh=dea10f95773803c35f1431b380542711\&oe=5EC4F33F, consulté le 30 janvier 2020.

25. Elisha Coles, An English Dictionary, Londres : Samuel Crouch, 1676, F1v.

26. Réplique de Tybalt affichée sur les écrans au-dessus de la scène lors de la représentation du 26 avril 2018 dans le théâtre national de Craiova.

27. Transcription de la représentation de Roméo et Juliette (2006), op. cit., p. 21.

28. Simon Buia, op.cit., http://www.romanianactors.com/wp-content/uploads/2018/04/ROMEO\%C5\%9EI-JULIETA-Mokwha-Repertory-Company-din-Seoul-Coreea-de-Sud-regia-Oh-Tae-SukPhotographer-Simion-Buia-www.romanianactors.com-4280307.jpg, consulté le 30 janvier 2020.

29. Festivalul International Shakespeare, Craiova, op. cit., https://scontent-mrs2-1.xx.fbcdn.net/ v/t31.0-8/30168023_2042995475952259_4315152559251729403_o.jpg?

_nc_cat $=105 \&$ _nc_ohc=NeQPSBfy0VIAX_UdBTD\&_nc_ht=scontent-

mrs2-1.xx\&oh=834c5d4c186190f6c520fe69ecd66d47\&oe=5ED58478, consulté le 30 janvier 2020.

30. Transcription de la représentation de Roméo et Juliette (2006), op. cit., p. 25.

31. Festivalul International Shakespeare, Craiova, op. cit., https://scontent-mrs2-1.xx.fbcdn.net/ v/t31.0-8/30425062_2042997909285349_6781383292019167673_o.jpg?

_nc_cat $=108 \&$ nc_ohc=i_FaWsGUYNYAX_LiaAf\&_nc_ht=scontent-

mrs2-1.xx\&oh=d169ae07d83902eefd7b239b120da3ac\&oe=5ED95522, consulté le 30 janvier 2020.

32. Entretien filmé par Sorin Cazacu lors de la conférence d'ESRA (European Shakespeare Research Association) "Viewing \& Reviewing: Shakespeare in Performance », Université de Craiova, 27 avril 2018, accessible en ligne sur: https://www.facebook.com/sorin.cazacu.7/ videos/1666678753412829/, consulté le 22 janvier 2019, 06:45-08:25.

33. Simon Buia, op.cit., http://www.romanianactors.com/wp-content/uploads/2018/04/ROMEO\%C5\%9EI-JULIETA-Mokwha-Repertory-Company-din-Seoul-Coreea-de-Sud-regia-Oh-Tae-SukPhotographer-Simion-Buia-www.romanianactors.com-4280484.jpg, consulté le 30 janvier 2020.

34. Transcription de la représentation de Roméo et Juliette (2006), op. cit., p. 17.

35. Festivalul International Shakespeare, Craiova, op. cit., https://scontent-mrs2-1.xx.fbcdn.net/ v/t31.0-8/30425504_2042995175952289_8243237012463349270_o.jpg?

_nc_cat=100\&_nc_ohc=I_1QxITEYgwAX_Rf5kG\&_nc_ht=scontent-

mrs2-1.xx\&oh=2aaac8e2cb4f2f1fa606c076765a90dd\&oe=5E8E0BE9, consulté le 30 janvier 2020.

36. Keith Pratt et Richard Rutt, Korea: A Historical and Cultural Dictionary, Londres et New York, Routledge, 1999, p. 127-128. 
37. Entretien lors de la conférence d'ESRA (European Shakespeare Research Association) «Viewing \& Reviewing : Shakespeare in Performance », Université de Craiova, 27 avril 2018.

38. Festivalul International Shakespeare, Craiova, op. cit., https://scontent-mrs2-2.xx.fbcdn.net/ v/t31.0-8/30171293_2042997912618682_1620116742586879442_o.jpg?

_nc_cat $=101 \&$ _nc_ohc $=$ AvNYvJhoEA4AX8gLW7C\&_nc_ht=scontent-

mrs2-2.xx\&oh=46333bf27ff29188f604d9d06074ddae\&oe=5ED71D49, consulté le 30 janvier 2020.

39. Entretien filmé par Sorin Cazacu lors de la conférence d'ESRA (European Shakespeare Research Association) "Viewing \& Reviewing: Shakespeare in Performance ", Université de Craiova, 27 avril 2018, accessible en ligne sur: https://www.facebook.com/sorin.cazacu.7/ videos/1666678753412829/, 20:40-21:30.

40. La destruction du mur a été ajoutée quelques semaines seulement après l'élection présidentielle aux États-Unis.

41. Simon Buia, op.cit., http://www.romanianactors.com/wp-content/uploads/2018/04/ROMEO\%C5\%9EI-JULIETA-Mokwha-Repertory-Company-din-Seoul-Coreea-de-Sud-regia-Oh-Tae-Suk-

Photographer-Simion-Buia-www.romanianactors.com-4281167.jpg, consulté le 30 janvier 2020.

42. Entretien filmé par Sorin Cazacu lors de la conférence d'ESRA, op. cit., 11:40-14:15.

43. Ibid.

44. Je suis redevable à Janice Valls-Russel pour cette référence.

45. Entretien filmé par Sorin Cazacu lors de la conférence d'ESRA, op. cit., 05:53-11:05.

46. Les tensions sont réapparues suite à des tirs de missiles effectués par la Corée du Nord en novembre 2019.

Philippe Mesmer, «La Corée du Nord reprend les provocations », Le Monde, 30 novembre 2019, accessible en ligne sur: https://www.lemonde.fr/international/article/2019/11/30/la-coree-dunord-reprend-les-provocations_6021161_3210.html, consulté le 30 janvier 2020.

Je suis redevable à Jean-Christophe Mayer pour cette référence.

\section{RÉSUMÉS}

Depuis 1999, la représentation du Roméo et Juliette d'Oh Tae-Suk a été interprétée dans plusieurs pays. Des changements importants sont survenus depuis la première, mais certains éléments ont perduré : la Nourrice s'adresse à son entourage avec des noms d'animaux et les acteurs portent des masques d'animaux lors de la scène du bal. L'imaginaire animal ainsi que la référence à l'astrologie (signes du zodiaque) permettent d'identifier et de caractériser les personnages sur scène, élément de la culture coréenne qu'Oh Tae-Suk ajoute à l'intrigue shakespearienne. Cependant, ce n'est pas suffisant pour résumer l'esthétique d'Oh Tae-Suk. D'autres aspects essentiels contribuent à faire de l'histoire des amants maudits un thème universel, comme l'absence de noms propres et la présence de masques animaliers. Les effets visuels et sonores sur scène remplacent les mots présents dans la tragédie shakespearienne, présentant ainsi l'amour entre deux êtres comme transcendant le langage. Ce compte rendu critique démontre comment l'imaginaire animal tel qu'utilisé dans la représentation du Roméo et Juliette d'Oh Tae-Suk, qui a eu lieu en 2018 au théâtre national de Craiova en Roumanie, fait partie intégrante de l'esthétique du metteur en scène sud-coréen. Grâce à l'alliance d'une dramaturgie " européenne » et d'éléments culturels coréens, Oh Tae-Suk répand un message d'espoir pour une paix universelle à l'échelle internationale adaptant une tragédie shakespearienne que sa troupe de théâtre expose aux quatre coins du monde. 
Oh Tae-Suk's production of Romeo and Juliet has been touring internationally since 1999. If major changes have occurred since the premiere, some elements have remained the same: the Nurse addresses the characters on stage uttering animal names, and the actors wear animal masks during the ball scene. Together with several references to astrology, the use of animal imagery to identify and define characters is a Korean twist Oh Tae-Suk adds to the Shakespearean plot. However, this argument alone would not fully encapsulate the South-Korean director's aesthetic. Other crucial aspects contribute to the presentation of the story of the star-crossed lovers as a universal one, such as the absence of proper names and the presence of animal masks. On stage, sound and visual elements replace the names and words of the Shakespearian tragedy, thus showing that love gives wings to fly whatever the language spoken. This theatre performance review essay aims at showing how animal imagery in Oh Tae-Suk's 2018 production of Romeo and Juliet performed at the National Theatre of Craiova, Romania, is part and parcel of the SouthKorean director's own aesthetic. Blending European dramaturgy and Korean cultural elements, he spreads a message of hope for a universal and international peace through this Shakespearean tragedy as his theatre company takes the show around the world.

\section{INDEX}

Mots-clés : adaptation, animaux légendaires, concepts universels, environnement, folklore coréen, représentation théâtrale, symbolisme animal.

Keywords : adaptation, animal symbolism, animal imagery, environment, Korean folklore, legendary animals, performance, universal concepts.

\section{AUTEUR}

\section{CHARLÈNE CRUXENT}

Université Paul-Valéry Montpellier 3 